\title{
Dissipative charge transport in diffusive superconducting double-barrier junctions
}

\author{
E. V. Bezuglyi, ${ }^{1,}{ }^{*}$ E. N. Bratus', ${ }^{1}$ and V. S. Shumeiko ${ }^{2}$ \\ ${ }^{1}$ B. Verkin Institute for Low Temperature Physics and Engineering, UA-61103 Kharkov, Ukraine \\ ${ }^{2}$ Chalmers University of Technology, SE-41296 Göteborg, Sweden \\ (Received 14 February 2011; revised manuscript received 23 March 2011; published 23 May 2011)
}

\begin{abstract}
We solve the coherent multiple Andreev reflection (MAR) problem and calculate current-voltage characteristics (IVCs) for Josephson SINIS junctions, where $\mathrm{S}$ are local-equilibrium superconducting reservoirs, I denotes tunnel barriers, and $\mathrm{N}$ is a short diffusive normal wire, the length of which is much smaller than the coherence length, and the resistance is much smaller than the resistance of the tunnel barriers. The charge transport regime in such junctions qualitatively depends on a characteristic value $\gamma=\tau_{d} \Delta$ of relative phase shifts between the electrons and retroreflected holes accumulated during the dwell time $\tau_{d}$. In the limit of small electron-hole dephasing $\gamma \ll 1$, our solution recovers a known formula for a short mesoscopic connector extended to the MAR regime. At large dephasing, the subharmonic gap structure in the IVC scales with $\gamma^{-1}$, which thus plays the role of an effective tunneling parameter. In this limit, the even gap subharmonics are resonantly enhanced, and the IVC exhibits portions with negative differential resistance.
\end{abstract}

DOI: 10.1103/PhysRevB.83.184517

PACS number(s): 74.50.+r, 74.45.+c

\section{INTRODUCTION}

Accurate theoretical description of nonequilibrium charge transport in Josephson junctions is an important and active research field. The concept of multiple Andreev reflections ${ }^{1}$ (MAR) is a universal framework explaining the nature of dissipative current in different types of junctions. The quasiparticles injected in the junction at applied voltage smaller than the superconducting energy gap $e V<2 \Delta$ can only escape into reservoirs at zero temperature by multiple traversing the junction due to repeated Andreev reflections, each time gaining energy $e V$. Such a process generates coherent transfer of multiple electron charge $n e=2 \Delta / V$ across the junction. Such a mechanism is important at small temperatures when the single-particle tunneling is exponentially weak and the Andreev transport becomes dominant. The most complete quantitative MAR theory has been developed for ballistic contacts. The central element here is the solution for a single conducting channel with given transmissivity, which enters the sum over conducting channels in the net current. Various approaches for constructing such a solution have been developed based on the scattering theory, tunneling Hamiltonian, and quasiclassical Green's functions. ${ }^{2-6}$

Extension of the theory has been suggested for disordered weak links, e.g., diffusive constrictions and superconductorinsulator-superconductor (SIS) tunnel junctions with disordered insulating layers. In this case, the summation over the channels is performed by taking into account the distribution of random transmission eigenvalues for corresponding structure in the normal state. ${ }^{7-9}$ This method, however, is only valid for short superconducting weak links, in which the dwell time, i.e., the time of quasiparticle diffusion along the whole MAR staircase, is small on a quantum time scale defined by the inverse order parameter $\Delta^{-1}$. In junctions with large dwell time, e.g., long superconductor-normal metalsuperconductor (SNS) junctions and double-barrier SINIS junctions containing Breit-Wigner resonances, the method does not work because of strong energy dispersion of the scattering amplitudes. The electronlike and holelike quasiparticles acquire different scattering phases during propagation through the junction (electron-hole dephasing), which results in the single-channel MAR current being dependent on the scattering amplitudes rather than the scattering coefficients. ${ }^{10-12}$ Thus, the distribution of transmission eigenvalues becomes irrelevant and is to be replaced by the statistics of scattering amplitudes, which is generally unknown except for some particular cases. ${ }^{13}$

In order to investigate the MAR problem in diffusive junctions with large electron-hole dephasing, one has to directly solve quasiclassical Keldysh-Green's function equations. ${ }^{14}$ This task, however, is technically demanding because a nonlinearity of equations and nonstationary character of the MAR problem lead to hardly tractable two-dimensional infinite chains of Green's function harmonics. So far, the problem has been analytically solved for superconducting constrictions, ${ }^{15}$ and for the opposite case of very long SNS and SINIS junctions, where the Josephson current is completely suppressed and an incoherent MAR theory can be formulated ${ }^{16,17}$ analogous to the one for ballistic junctions. ${ }^{18}$ A perturbative scheme for a superconducting film interrupted by a tunnel junction has been suggested; ${ }^{19}$ however, no general methods to analytically treat the problem in SNS junctions of intermediate lengths exist up to now, and even a numerical solution presents a difficult and time-consuming task. ${ }^{20}$

In this paper, we present a study of the junction for which the coherent MAR problem can be relatively easily solved and fully investigated both analytically and numerically for the entire range from weak to strong electron-hole dephasing. We consider a SINIS junction with opaque NIS interfaces having equal resistances $R$ much larger than the resistance $R_{N}$ of the diffusive normal wire, the length $2 d$ of which is supposed to be much smaller than the coherence length $\sqrt{\mathcal{D} / \Delta}$ ( $\mathcal{D}$ is the diffusion coefficient, $\hbar=1$ ). In such junctions, the dwell time $\tau_{d}$ is characterized by the parameter ${ }^{16,21-23}$

$$
\gamma=\tau_{d} \Delta=\frac{R}{R_{N}} \frac{\Delta}{E_{\mathrm{Th}}},
$$

where $E_{\mathrm{Th}}=\mathcal{D} /(2 d)^{2}$ is the Thouless energy. This parameter defines the amplitude of the Josephson current and the magnitude of the minigap in the energy spectrum within the 
normal wire $\Delta_{g} \sim \Delta /(1+\gamma)$. In short junctions, the Thouless energy is large compared to $\Delta$, therefore, the parameter $\gamma$ may have arbitrary value depending on the junction length and transparency. Correspondingly, the value of the minigap may vary between 0 and $\Delta$, reflecting the crossover from large to small electron-hole dephasing.

We construct the MAR solution of the Keldysh-Green's function equations and evaluate the dc current for arbitrary $\gamma$ by means of the second-order recurrences similar to that for ballistic structures. ${ }^{4,5}$ For the case of small electron-hole dephasing $(\gamma \ll 1)$, we show that the current is given by a general formula for mesoscopic connector ${ }^{24}$ [see Eq. (10) below], i.e., the average of the result for the single channel ${ }^{4,5}$ over the distribution of transmission eigenvalues for a doublebarrier normal diffusive structure. ${ }^{25}$ This result coincides with the result of Ref. 9 and it corresponds to a broad Breit-Wigner resonance in the single channel. In the opposite case of large electron-hole dephasing $\gamma \gg 1$, the current can be explicitly presented as a sum of multiparticle tunnel currents, which scale with the effective tunneling parameter $\gamma^{-1}$.

The structure of the paper is as follows. We formulate basic equations and construct analytical solutions in Sec. II. In Sec. III, we present and discuss the results of numerical calculation of the current-voltage characteristics (IVCs). The multiparticle currents in the limit $\gamma \gg 1$ are calculated analytically in Sec. IV, which also includes evaluation of the excess current.

\section{BASIC EQUATIONS AND SOLUTION}

We start our quantitative consideration with the equation for the Keldysh-Green's function $\breve{G}\left(x, t_{1}, t_{2}\right)$ in the normal wire $(-d<x<d)$,

$$
\left[\sigma_{z} \hat{E}, \check{G}\right]=i \mathcal{D} \partial_{x}\left(\check{G} \partial_{x} \check{G}\right), \quad \check{G}^{2}=1, \quad \check{G}=\left(\begin{array}{cc}
\hat{g}^{R} & \hat{G}^{K} \\
0 & \hat{g}^{A}
\end{array}\right) .
$$

Here, $\hat{g}^{R, A}$ are the retarded and advanced Green's functions, $\hat{f}$ is the matrix distribution function, $\hat{G}^{K}=\hat{g}^{R} \hat{f}-\hat{f} \hat{g}^{A}$, and the kernel of the energy operator $\hat{E}$ is $E\left(t_{1}, t_{2}\right)=i \partial_{t_{1}} \delta\left(t_{1}-t_{2}\right)$. All products in Eq. (2) are time convolutions: $(A B)\left(t_{1}, t_{2}\right)=$ $\int d t A\left(t_{1}, t\right) B\left(t, t_{2}\right)$. The electric current is defined as

$$
I(t)=\left(\pi g_{N} / 4 e\right) \operatorname{Tr} \tau_{K}\left(\check{G} \partial_{x} \check{G}\right)(t, t), \quad \tau_{K}=\sigma_{z} \tau_{x}
$$

where $g_{N}$ is the conductance of the normal wire per unit length, and the $\sigma$ and $\tau$ matrices operate in the Nambu and the Keldysh space, respectively. At the tunnel barriers $x= \pm d$, we apply the Kupriyanov-Lukichev boundary conditions ${ }^{26}$

$$
g_{N}\left(\check{G} \partial_{x} \check{G}\right)_{ \pm d}= \pm(2 R)^{-1}\left[\check{G}_{ \pm d}, \check{G}_{R, L}\right]
$$

The equilibrium Keldysh-Green's functions $\check{G}_{R, L}$ in the right and left superconducting electrodes are constructed with the local-equilibrium Green's and distribution functions

$$
\begin{gathered}
\hat{g}_{R, L}=\sigma_{z} u\left(\epsilon_{ \pm}\right)+i \exp \left( \pm i \sigma_{z} e V t\right) \sigma_{y} v(E) \\
\hat{f}_{R, L}=f\left(\epsilon_{ \pm}\right), \quad f(E)=\tanh \frac{E}{2 T}, \quad \epsilon_{ \pm}=E \pm \sigma_{z} \frac{e V}{2}
\end{gathered}
$$

$$
u^{R, A}(E)=\frac{E}{\xi}, \quad v^{R, A}(E)=\frac{\Delta}{\xi}, \quad \xi^{R, A}=\sqrt{(E \pm i 0)^{2}-\Delta^{2}},
$$

given in the $(E, t)$ representation: $A(E, t)=\int d \tau e^{i E \tau} A(t+$ $\tau / 2, t-\tau / 2)$. In Eqs. (5), we use the antisymmetric gauge of the superconducting phase $\phi_{R}=-\phi_{L}=e V t$, satisfying the Josephson relation $\phi=\phi_{R}-\phi_{L}=2 e V t$.

Solution of Eqs. (2)-(5), being generally difficult, essentially simplifies in short junctions with opaque barriers. Averaging Eq. (2) along the wire and using Eqs. (4) and (1), we get

$$
2 \gamma\left[\sigma_{z} \hat{E}, \check{G}\right]=i \Delta\left(\left[\check{G}_{d}, \check{G}_{R}\right]+\left[\check{G}_{-d}, \check{G}_{L}\right]\right),
$$

where $\bar{G}$ denotes spatially averaged value of $\check{G}$. In the tunnel limit $R \gg R_{N}$, the Keldysh-Green's functions are approximately spatially homogeneous within the normal wire ${ }^{21,23}$ $\overline{\breve{G}} \approx \check{G}_{d} \approx \check{G}_{-d}$. Thus, denoting these quantities by a single notation $\check{G}$, we arrive at the commutator equation

$$
[\check{A}, \check{G}]=0, \quad \check{A}=\check{G}_{+}-i \sigma_{z} \tau_{d} \hat{E}, \quad \check{G}_{ \pm}=\frac{1}{2}\left(\check{G}_{R} \pm \check{G}_{L}\right) .
$$

A similar approach has been used in analysis of current transport in a NINIS structure. ${ }^{27}$ Following Refs. 27 and 28, we find a physically relevant solution of Eq. (7) satisfying the normalization condition $\check{G}^{2}=1$ in Eq. (2),

$$
\check{G}=\frac{\check{A}}{\sqrt{\check{A}^{2}}}=\frac{1}{\pi} \int_{-\infty}^{\infty} d \lambda \check{K}(\lambda), \quad \check{K}(\lambda)=(\check{A}+i \lambda)^{-1} .
$$

By applying Eqs. (4) to (3) and using (8), we symmetrize the quantity $I(t)$ with respect to the left and right reservoirs,

$$
I(t)=\int_{-\infty}^{\infty} \frac{d \lambda}{8 e R} \operatorname{Tr} \tau_{K}\left[\check{K}(\lambda), \check{G}_{-}\right](t, t) .
$$

The structure of the matrix current $\left[\check{K}(\lambda), \check{G}_{-}\right]$in Eq. (9) is quite similar to the solution of the MAR problem for a single ballistic channel with the transparency $D=\left(\lambda^{2}+1\right)^{-1}$ given in Ref. 15 and differs from the latter by an additional term $-i \sigma_{z} \tau_{d} \hat{E}$ in the matrix $\breve{K}$, which describes the electron-hole dephasing during the dwell time $\tau_{d}$. If this effect is negligibly small, $\gamma \rightarrow 0$, Eq. (9) rewritten in terms of the functions $G_{L, R}$ and the transparency variable $D$ can be transformed to the known formula for a short connector ${ }^{24}$ generalized to the nonstationary case of voltage-biased SINIS junction,

$$
\begin{gathered}
I(t)=\frac{\pi}{8 e R} \int_{0}^{1} d D \operatorname{Tr} \tau_{K} \frac{D \rho(D)\left[\check{G}_{L}, \check{G}_{R}\right]}{1+\frac{D}{4}\left(\left\{\check{G}_{L}, \check{G}_{R}\right\}-2\right)}(t, t), \\
\rho(D)=\frac{1}{\pi D^{3 / 2} \sqrt{1-D}}, \quad \int_{0}^{1} D \rho(D) d D=1 .
\end{gathered}
$$

The fact that the MAR current in this limit is given by a convolution of nonresonant single-channel current with the transparency distribution $\rho(D)$ for a double-barrier potential ${ }^{25}$ (see also Refs. 8 and 29) is consistent with a wide resonance 
in the single channel. This result justifies the method and the result of Ref. 9. We note that, in the static limit $\dot{\phi}=0$, Eq. (8) reproduces the result ${ }^{21,23}$ of a direct solution of Usadel equations

$$
\hat{g}=\frac{\sigma_{z} E+i \sigma_{y} \Delta(E, \phi)}{\sqrt{E^{2}-\Delta^{2}(E, \phi)}},
$$

where $\Delta(E, \phi)=\Delta \cos (\phi / 2)[1-i \gamma / v(E)]^{-1}$.

In the general case of arbitrary $\gamma$, calculation of the matrix $\check{K}$ in Eq. (8) can be performed by expanding all quantities over the harmonics of $e V: A(E, t)=\sum_{m} A(E, m) e^{-i m e V t}$. In this representation, the time-averaged (dc) current reads as

$$
I=\int_{-\infty}^{\infty} \int_{-\infty}^{\infty} \frac{d \lambda d E}{16 \pi e R} \sum_{m} \operatorname{Tr} \check{K}(\lambda, E, m)\left[\check{G}_{-}(E,-m), \tau_{K}\right],
$$

and the local-equilibrium functions contain only three harmonics, $m=0, \pm 1$. In Eq. (13), we rearranged the factors in the integrand using the fact that time averaging is equivalent to the trace in the time domain. After some algebra, we find the function $\breve{G}_{+}=\sigma_{z} G_{0}^{+} \delta_{m, 0}+i \sigma_{y} G_{1}^{+} \delta_{|m|, 1}$ and the commutator $\left[\check{G}_{-}, \tau_{K}\right]=\sigma_{z} G_{0}^{-} \delta_{m, 0}+i \sigma_{y} G_{1}^{-} m \delta_{|m|, 1}$, where

$$
\begin{gathered}
G_{0}^{+}=\frac{1}{2}\left[i\left(\bar{N}_{+}+\bar{N}_{-}\right)+N_{+} F_{+}+N_{-} F_{-}\right], \\
G_{0}^{-}=\tau_{z}\left(f_{+} N_{+}-f_{-} N_{-}\right)+i \tau_{y}\left(N_{+}-N_{-}\right), \\
G_{1}^{+}=\frac{1}{2}(i \bar{M}+M F), \quad G_{1}^{-}=i \bar{M} \tau_{x}+M f \\
F=\tau_{z}+2 f \tau_{+}, \quad \tau_{+}=(1 / 2)\left(\tau_{x}+i \tau_{y}\right), \\
N=\operatorname{Re} u^{R}, \quad M=\operatorname{Re} v^{R}, \quad \bar{N}=\operatorname{Im} u^{R}, \quad \bar{M}=\operatorname{Im} v^{R} .
\end{gathered}
$$

Here and in the following, the lower indices \pm denote the energy shift by $\pm e V / 2$. The function $N(E)$ is the BCS density of states (normalized over its value in the normal metal), which turns to zero at $|E|<\Delta$ along with the function $M(E)$, while the functions $\bar{N}(E)$ and $\bar{M}(E)$ vanish outside the energy gap. This leads to the following expression for the dc current:

$$
\begin{aligned}
I=\int_{-\infty}^{\infty} d E J(E), \quad J(E)=\int_{-\infty}^{\infty} \frac{d \lambda}{16 \pi e R} j(E, \lambda), \\
j=j_{0}+j_{1}+j_{-1}, \\
j_{0}=\operatorname{Tr} \check{K}(E, 0) \sigma_{z} G_{0}^{-}, \quad j_{ \pm 1}= \pm \operatorname{Tr} \check{K}(E, \mp 1) i \sigma_{y} G_{1}^{-} .
\end{aligned}
$$

Here, $J(E)$ is the current spectral density, whereas the quantity $j(E, \lambda)$ can be interpreted as a generalized spectral density depending on the auxiliary parameter $\lambda$.

According to Eq. (8), the matrix $\check{K}(\lambda)$ obeys the equation $(\check{A}+i \lambda) \check{K}(\lambda)=1$; in the $(E, m)$ representation, it has the form

$$
\begin{aligned}
& \sum_{m^{\prime}} G_{+}\left[E+\left(m-m^{\prime}\right) e V / 2, m^{\prime}\right] K\left(E-e V m^{\prime} / 2, m-m^{\prime}\right) \\
& \quad+i\left[\lambda-\sigma_{z} \tau_{d}(E+e V m / 2)\right] K(E, m)=\delta_{m, 0}
\end{aligned}
$$

(we omit the "check" on top of the $4 \times 4$ matrices), where the sum actually contains only three nonzero terms with $m^{\prime}=$ $0, \pm 1$. Introducing the quantity $K_{m}(E)=K(E+m e V / 2, m)$, we obtain the $4 \times 4$ matrix recurrence relation

$$
\begin{aligned}
& \left(H_{m}+i \lambda\right) K_{m}+h_{m} K_{m-1}+h_{m+1} K_{m+1}=\delta_{m, 0}, \\
& H_{m}=\sigma_{z} Q_{m}, \quad Q_{m}=G_{0}^{+}\left(E_{m}\right)-i \tau_{d} E_{m}, \\
& h_{m}=i \sigma_{y} q_{m}, \quad q_{m}=G_{1}^{+}\left(E_{m-1 / 2}\right), \quad E_{m}=E+m e V .
\end{aligned}
$$

Solution of Eq. (20) can be found by the matrix version of the chain fractions formalism ${ }^{4,5}$ using the ansatz

$$
K_{m}= \begin{cases}S_{m} S_{m-1} \ldots S_{1} K_{0}, & m>0 \\ P_{m} P_{m+1} \ldots P_{-1} K_{0}, & m<0 .\end{cases}
$$

Recurrence relations for the "matrix chain fractions" $S_{m}$ and $P_{m}$ with the boundary conditions $S_{m} \rightarrow 0$ at $m \rightarrow \infty$ and $P_{m} \rightarrow 0$ at $m \rightarrow-\infty$ follow from Eqs. (20) and (21) at $m \neq 0$,

$$
\begin{aligned}
& S_{m}=-\left(H_{m}+i \lambda+h_{m+1} S_{m+1}\right)^{-1} h_{m}, \\
& P_{m}=-\left(H_{m}+i \lambda+h_{m} P_{m-1}\right)^{-1} h_{m+1} .
\end{aligned}
$$

At $m=0$, we obtain a nonuniform equation, the solution of which is $K_{0}(E)=\left(H_{0}+i \lambda+h_{0} P_{-1}+h_{1} S_{1}\right)^{-1}$. Thus, the functions $K(E, m)$ in Eq. (19) read as

$$
\begin{gathered}
K(E, 0)=K_{0}(E), \quad K(E, 1)=S_{1}\left(E_{-}\right) K_{0}\left(E_{-}\right), \\
K(E,-1)=P_{-1}\left(E_{+}\right) K_{0}\left(E_{+}\right) .
\end{gathered}
$$

The $4 \times 4$ recurrences in Eqs. (22) can be reduced to the $2 \times 2$ form in the Keldysh space. Indeed, assuming $S_{m}=$ $-\sigma_{x} \bar{S}_{m}$ and $P_{m}=-\sigma_{x} \bar{P}_{m}$, we arrive at the recurrences for $\bar{S}$ and $\bar{P}$, which are diagonal in the Nambu space, 24

$$
\begin{aligned}
& \bar{S}_{m}=\left(Q_{m}-i \lambda \sigma_{z}-q_{m+1} \bar{S}_{m+1}^{\prime}\right)^{-1} q_{m}, \\
& \bar{P}_{m}=\left(Q_{m}-i \lambda \sigma_{z}-q_{m} \bar{P}_{m-1}^{\prime}\right)^{-1} q_{m+1},
\end{aligned}
$$

where the prime sign denotes the change of the sign of the $\sigma_{z}$ component of the matrix. Then, the function $K_{0}$ is also found to be diagonal in the Nambu space,

$$
K_{0}=\sum_{\sigma= \pm 1} \frac{1}{2}\left(\sigma+\sigma_{z}\right)\left(Q_{0}+i \lambda \sigma-q_{0} \bar{P}_{-1}^{\sigma}-q_{1} \bar{S}_{1}^{\sigma}\right)^{-1} .
$$

The functions $\bar{S}^{\sigma}$ and $\bar{P}^{\sigma}$ satisfy Eqs. (24) in which $\sigma_{z}$ is replaced by the scalar $\sigma$. Then, introducing the quantities $s_{m}(\lambda)$ and $p_{m}(\lambda)$ according to $\bar{S}_{m}^{\sigma}=s_{m}(\sigma \lambda)$ and $\bar{P}_{m}^{\sigma}=p_{m}(\sigma \lambda)$, we arrive at the $2 \times 2$ recurrences for the Keldysh matrices

$$
\begin{aligned}
& s_{m}=\left[Q_{m}+i \lambda(-1)^{m}-q_{m+1} s_{m+1}\right]^{-1} q_{m}, \\
& p_{m}=\left[Q_{m}+i \lambda(-1)^{m}-q_{m} p_{m-1}\right]^{-1} q_{m+1} .
\end{aligned}
$$

In Eq. (25), rewritten through the matrices $s_{m}$ and $p_{m}$, we can replace $\sigma \lambda \rightarrow \lambda$, which does not change the result of the integration over $\lambda$ in Eq. (17); as the result, only the term proportional to $\sigma_{z}$ survives in Eq. (25):

$$
K_{0}=\sigma_{z} K, \quad K=\left[Q_{0}+i \lambda-q_{0} p_{-1}(\lambda)-q_{1} s_{1}(\lambda)\right]^{-1} .
$$

By combining Eqs. (19), (23), and (27), and shifting the energy in $j_{ \pm 1}$ by $\mp e V / 2$, which holds the result of integration over $E$ 


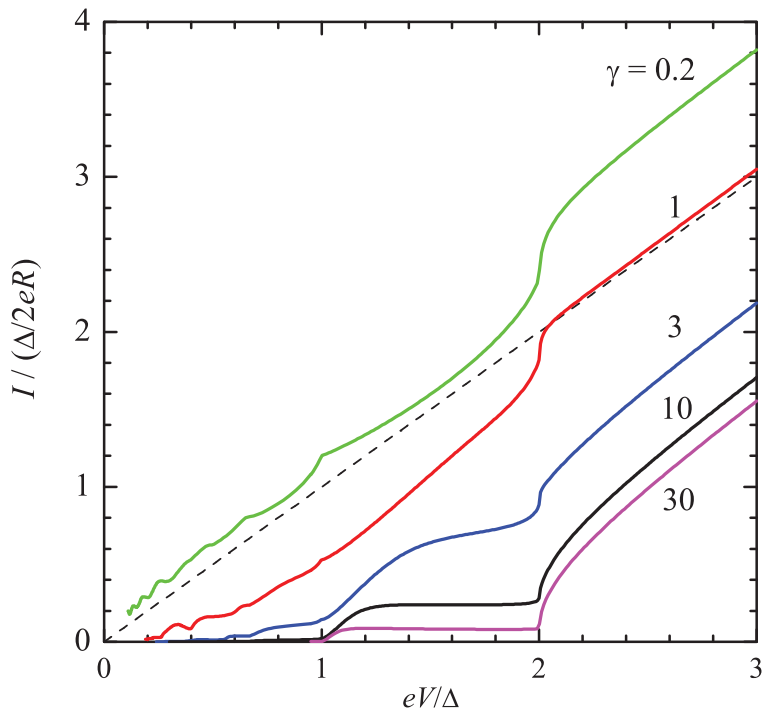

FIG. 1. (Color online) Current-voltage characteristics of a short diffusive SINIS junction at different values of the parameter $\gamma$.

in Eq. (17) unchanged, we obtain current spectral densities 28

$$
\begin{gathered}
j_{0}=2 \operatorname{Tr}_{\tau} K(E, \lambda) G_{0}^{-}(E), \\
j_{1}=-2 \operatorname{Tr}_{\tau} p_{-1}(E, \lambda) K(E, \lambda) G_{1}^{-}\left(E_{-}\right), \\
j_{-1}=2 \operatorname{Tr}_{\tau} s_{1}(E, \lambda) K(E, \lambda) G_{1}^{-}\left(E_{+}\right) .
\end{gathered}
$$

\section{NUMERICAL RESULTS}

Now we proceed with the numerical analysis of the dc current using Eqs. (26)-(28) and (17). The results obtained for a wide range of values of the parameter $\gamma$ and at zero temperature are shown in Figs. 1 and 2. As expected, at small electron-hole dephasing $\gamma=0.2$, the IVC is close to the results ${ }^{8,9}$ found from Eqs. (10) and (11). In this case, the IVC consists of concave portions between weakly pronounced features (steps and peaks) and reveals the excess current at large voltages. Since the edges $\pm \Delta_{g}$ of the minigap $\Delta_{g} \approx 0.8 \Delta$ are close to the edges $\pm \Delta$ of the superconducting energy gap, the existence of such a large minigap does not distort the periodicity of the IVC features, the positions of which approximately coincide with the gap subharmonics $e V=2 \Delta / n, n=1,2, \ldots$.

As the junction transparency decreases (i.e., $\gamma$ increases), the excess current also decreases, approaching zero at $\gamma \approx 1$, and then becomes negative (deficit current). Simultaneously, the peaks in the IVC almost vanish, and the subharmonic gap structure at small $e V$ and $\gamma=1-3$ becomes somewhat chaotic (see Fig. 2). This is due to the interplay of the contributions of MAR trajectories touching the superconducting gap edges and the edges of the minigap $\Delta_{g} \approx(0.25-0.5) \Delta$. Then, at large $\gamma=10-30$, the IVC features become regular again and their positions exactly correspond to the gap subharmonics. In this case, the minigap is small, $\Delta_{g} \approx 0.03-0.1$, and therefore affects the MAR trajectories touching the superconducting gap edges with even number of steps only. The enhanced density of states in the vicinity of the minigap increases the transmissivity of these MAR chains; this leads to anomalous enhancement of the magnitude of the dc current just above

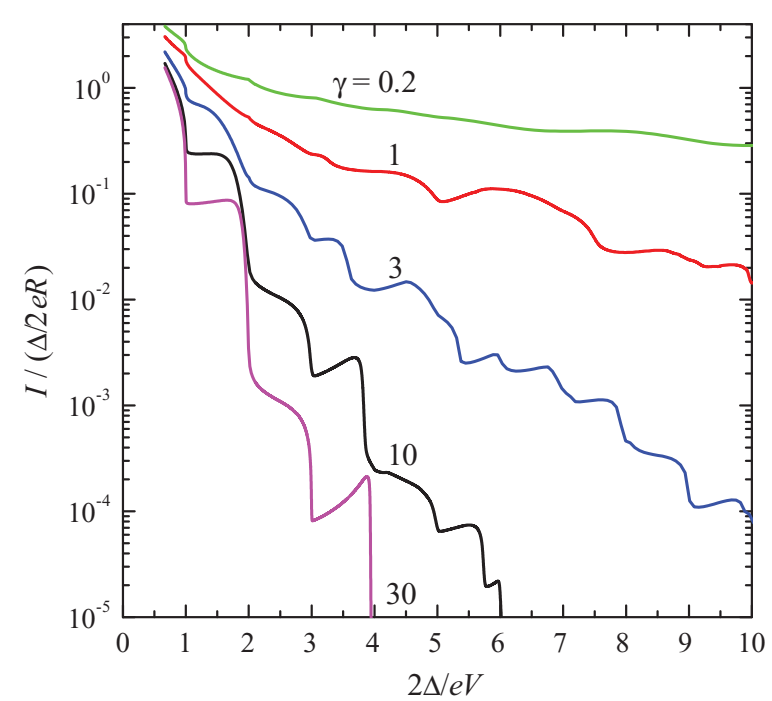

FIG. 2. (Color online) Current vs inverse voltage in logarithmic scale.

the even gap subharmonics $n=2 k$. As $k$ increases, this resonance effect becomes more pronounced and leads to the appearance of the IVC portions with negative differential resistance, as seen in Fig. 2. The current spectral density $J(E)$ shown in Fig. 3 at $2 \Delta / n<e V<2 \Delta /(n-1), n=1-4$, has the form of $n$ main equal peaks, which acquire a resonant shape for $n=2 k$ [Figs. 3(b) and 3(d)], in accordance with the above-mentioned anomalous transmissivity of even MAR chains. Small footprints of these resonances are visible in the spectral density of the odd-particle currents with $n=2 k-1$ [Figs. 3(a) and 3(c)].

We note that, at $\gamma>1$ and $e V<\Delta$, the averaged IVC is well approximated by the dependence

$$
I(V)=\frac{0.18 \Delta^{2}}{e R \Delta_{g}} \gamma^{-2 \Delta / e V},
$$

which is similar to the result for the ballistic SIS structure ${ }^{30}$ with $\gamma^{-1}$ standing for the transparency $D$. Thus, at $\gamma>1$, the quantity $\gamma^{-1}$ plays the role of an effective parameter for multiparticle tunneling processes, which determines the value of the $n$-particle current of the order of $R^{-1} \gamma^{1-n}$. This conclusion is confirmed by asymptotic analysis of multiparticle currents (see below).

\section{ANALYTICAL RESULTS}

In this section, we present a detailed analytical discussion of the dc current. To this end, we consider Eqs. (26) as functional equations for the functions $S(E, \lambda)=s_{1} G_{1}^{+}\left(E_{+}\right)$ and $P(E, \lambda)=p_{-1} G_{1}^{+}\left(E_{-}\right)$:

$$
\begin{gathered}
(S, P)(E, \lambda)=\left[K^{0}(E \pm e V,-\lambda)\right. \\
\left.+\Pi^{S, P}(E \pm e V,-\lambda)\right]^{-1}, \\
K^{0}(E, \lambda)=G_{0}^{+}-i \tau_{d} E+i \lambda, \\
\Pi^{S, P}(E, \lambda)=-G_{1}^{+}\left(E_{ \pm}\right)(S, P)(E, \lambda) G_{1}^{+}\left(E_{ \pm}\right) .
\end{gathered}
$$


In terms of these functions, the contributions (28b) and (28c) of nonzero harmonics to the dc current read as

$$
\begin{gathered}
j_{ \pm 1}=\mp 2 \operatorname{Tr}_{\tau}(P, S) G_{1}^{+}\left(E_{\mp}\right) \widetilde{K}^{-1} G_{1}^{-}\left(E_{\mp}\right), \\
\widetilde{K}=K^{0}+\Pi^{P}+\Pi^{S} .
\end{gathered}
$$

Analysis of Eq. (30) shows that the matrices $K^{0}, P$, and $S$ possess certain symmetry properties with respect to the transformation $E \rightarrow-E, \lambda \rightarrow-\lambda: P^{1,+} \leftrightarrow-S^{1,+}, P^{z} \leftrightarrow$ $S^{z}, Z_{P} \leftrightarrow Z_{S}, Z_{K} \leftrightarrow Z_{K}$, where $Z_{K}=\operatorname{det} \tilde{K}, Z_{P}=\operatorname{det} P$, $Z_{S}=\operatorname{det} S$, and the upper indices $1, z$, and + denote $1-$, $\tau_{z^{-}}$, and $\tau_{+}$-matrix components, respectively. These relations allow us to exclude the antisymmetric terms that vanish under integration over $E$ and $\lambda$ in Eq. (17) and to write down the spectral densities Eqs. (28) in a compact form

$$
\begin{gathered}
j_{0}=-\frac{2}{Z_{K}}\left[2 \widetilde{K}^{z}\left(N_{+} f_{+}-N_{-} f_{-}\right)-\widetilde{K}^{+}\left(N_{+}-N_{-}\right)\right], \\
j_{1}+j_{-1}=-\frac{2}{Z_{K}} \bar{M}_{-}^{2}\left(P^{z} \widetilde{K}^{+}-P^{+} \widetilde{K}^{z}\right) .
\end{gathered}
$$

\section{A. Excess current at $e V \gg \Delta$}

We start with evaluation of the excess current $I^{\text {exc }}$ at large applied voltage and for arbitrary $\gamma$. This quantity is contributed by both the single-particle current and the two-particle Andreev current. Formally, $I^{\text {exc }}$ is the voltage-independent term in the asymptotic expression for the dc current $I=V / 2 R+$ $I^{\text {exc }}+O(\Delta / e V)$ at $e V \gg \Delta$. In the corresponding expansion of full current spectral density Eq. (35), we truncate the recurrences for $P$ and $S$, omitting in $j(E, \lambda)$ the combinations
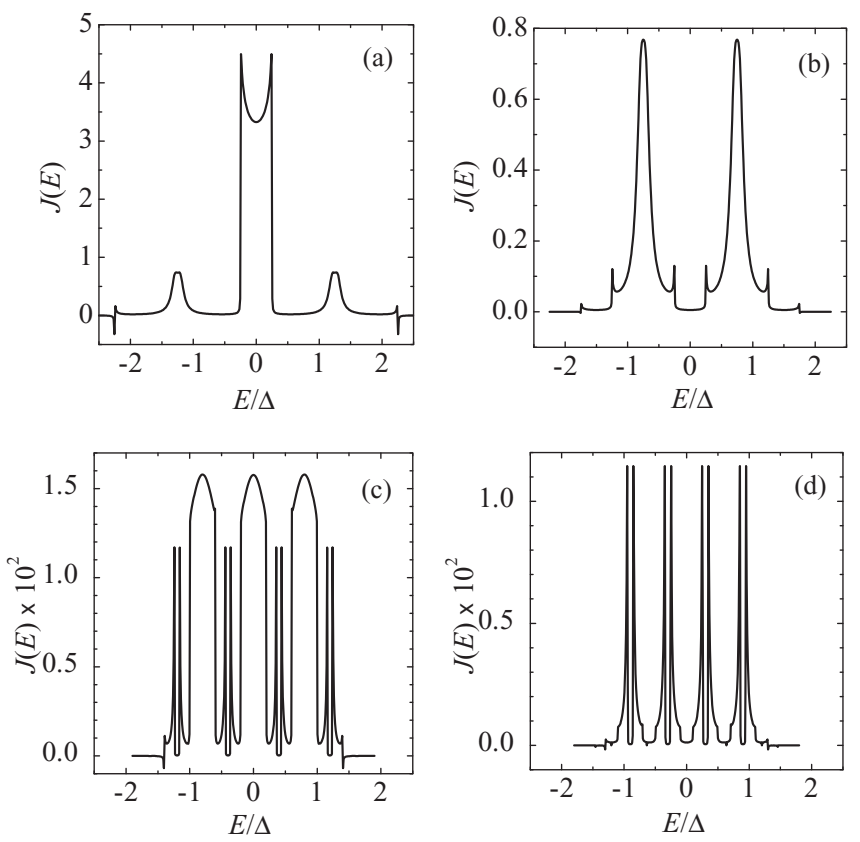

FIG. 3. Current spectral density $J(E)$ [normalized on $(2 e R)^{-1}$ ] at $\gamma=10$ and different applied voltages: (a) $e V=2.5 \Delta$, single-particle current; (b) $e V=1.5 \Delta$, two-particle current; (c) $e V=0.8 \Delta$, threeparticle current; (d) $e V=0.6 \Delta$, four-particle current. Visible are small contributions of higher even-particle processes into the spectral density of the odd-particle currents. of the functions $M_{\alpha}$ or $\bar{M}_{\alpha}$ with different energies (such as $M_{\alpha} M_{\beta}$ with $\beta \neq \alpha$ ), which turn to zero at $e V \rightarrow \infty$,

$$
\begin{aligned}
j(E, \lambda)= & \frac{Z_{P}}{Z_{K}}\left\{2 \bar{M}_{-1 / 2}^{2} L_{1 / 2,-3 / 2}-M_{-1 / 2}^{2}\left[L_{1 / 2,-1 / 2}\right.\right. \\
& \left.\left.\times\left(1+\frac{N_{-3 / 2}}{N_{-1 / 2}}\right)+L_{-1 / 2,-3 / 2}\left(1-\frac{N_{1 / 2}}{N_{-1 / 2}}\right)\right]\right\} \\
& -4 \frac{L_{1 / 2,-1 / 2}}{Z_{K}}, \\
& L_{\alpha \beta}=N_{\alpha} N_{\beta}\left(f_{\alpha}-f_{\beta}\right), \quad \alpha>\beta
\end{aligned}
$$

Here and in the following, the lower indices denote the energy shift, e.g., $N_{\alpha} \equiv N(E+\alpha e V)$. At zero temperature $(f=\operatorname{sgn} E)$, the factor $L_{\alpha \beta}$ is nonzero within the energy region

$$
\Delta-\alpha e V<E<-\Delta-\beta e V .
$$

Existence of this energy interval, in which $L_{\alpha \beta}=2 N_{\alpha} N_{\beta}$, imposes the following condition on the applied voltage:

$$
(\alpha-\beta) e V>2 \Delta ;
$$

otherwise, the function $L_{\alpha \beta}$ is identically zero. Since the convergence of integration over $E$ of all terms in curly brackets in Eq. (36) is ensured by the functions $M_{\alpha}$ or $\bar{M}_{\alpha}$ with $E_{\alpha} \sim \Delta$, the functions $N_{\beta}$ with different energies $(\beta \neq \alpha)$ can be approximated by their values in a normal metal $N_{\beta}=1$. By using these simplifications, we arrive at the following asymptotics of the dc current:

$$
\begin{gathered}
I=\frac{2 \Delta}{\pi e R} \int_{-\infty}^{\infty} d \lambda\left[\int_{1}^{e V / 2 \Delta} d x j_{1}(x, \lambda)+\int_{0}^{1} d x j_{2}(x, \lambda)\right], \\
j_{1}=\frac{x\left[(\lambda+y)^{2}+z^{2}-1\right]}{\left|\lambda^{2}-1+(z-i y)^{2}\right|^{2}}, \quad j_{2}=\frac{2 y_{1}}{\left|\lambda^{2}+1-\left(z_{1}-i y_{1}\right)^{2}\right|^{2}}, \\
y=2 \gamma x \sqrt{x^{2}-1}, \quad z=x+\sqrt{x^{2}-1}, \quad y_{1}=\sqrt{1-x^{2}}, \\
z_{1}=x\left(1+2 \gamma y_{1}\right) .
\end{gathered}
$$

By integrating over $\lambda$ in Eq. (40) and separating out the constant term, we obtain a general expression for the excess current:

$$
\begin{gathered}
I^{\mathrm{exc}}(\gamma)=\frac{\Delta}{e R}\left[\int_{1}^{\infty} d x j_{1}(x)+\int_{0}^{1} d x j_{2}(x)-1\right], \\
j_{1}(x)=\frac{\sqrt{2} x\left(\widetilde{T}+T^{2}-1\right)}{\widetilde{T} \sqrt{\widetilde{T}-A}}-1, \quad j_{2}(x)=\frac{2 \sqrt{2\left(1-x^{2}\right)}}{T_{1} \sqrt{T_{1}+A_{1}}}, \\
T^{2}=y^{2}+z^{2}, \quad \widetilde{T}^{2}=A^{2}+4 y^{2} z^{2}, \quad A=1+y^{2}-z^{2} \\
T_{1}^{2}=A_{1}^{2}+4 y_{1}^{2} z_{1}^{2}, \quad A_{1}=1+y_{1}^{2}-z_{1}^{2} .
\end{gathered}
$$

In the limit of small dephasing $\gamma \rightarrow 0$, when the integral over $x$ in Eq. (40) can be explicitly calculated, the substitution $D=\left(\lambda^{2}+1\right)^{-1}$ leads to the formula ${ }^{9}$

$$
\begin{aligned}
I^{\mathrm{exc}} & =\frac{\pi}{2 e R} \int_{0}^{1} d D \rho(D) I_{\mathrm{SIS}}^{\mathrm{exc}}(D)=0.53 \frac{\Delta}{e R}, \quad \gamma \ll 1 \\
I_{\mathrm{SIS}}^{\mathrm{exc}}(D) & =\frac{D^{2} \Delta}{\pi \mathcal{R}}\left[1-\frac{D^{2}}{2(1+\mathcal{R}) \sqrt{\mathcal{R}}} \ln \frac{1+\sqrt{\mathcal{R}}}{1-\sqrt{\mathcal{R}}}\right], \\
\mathcal{R} & =1-D
\end{aligned}
$$


which expresses the excess current through its value $I_{\text {SIS }}^{\mathrm{exc}}$ for a single ballistic channel ${ }^{31}$ averaged over the transparency distribution Eq. (11), in accordance with Eq. (10). In the opposite case $\gamma \gg 1, I^{\text {exc }}$ becomes negative (deficit current),

$$
I^{\mathrm{def}}=-\frac{2 \Delta}{3 e R}, \quad \gamma \gg 1 .
$$

\section{B. Multiparticle currents at large $\gamma$}

In the limit of large dephasing $\gamma \gg 1$, it is possible to express analytically the full current as a sum of contributions of $n$-particle tunneling processes. ${ }^{4}$ Here, we proceed with the asymptotic analysis of these partial contributions. First, we separate out the unity and the traceless components of the Keldysh matrices, e.g., $P=P^{1}+\hat{P}, \hat{P} \equiv \tau_{z} P^{z}+\tau_{+} P^{+}$:

$$
\begin{gathered}
\hat{\Pi}^{S, P}=-G_{ \pm 0}(\hat{S}, \hat{P}) G_{ \pm 0}, \quad \Pi^{S 1, P 1}=\tilde{M}_{ \pm 1 / 2}\left(S^{1}, P^{1}\right), \\
\widetilde{M}_{\alpha}=-\left[G_{1}^{+}\left(E_{\alpha}\right)\right]^{2}=\frac{1}{4}\left(\bar{M}_{\alpha}^{2}-M_{\alpha}^{2}\right),
\end{gathered}
$$

where $G_{ \pm m}=G_{1}^{+}\left(E_{ \pm} \pm m e V\right)$. By introducing the notations

$$
\begin{aligned}
& \hat{K}_{m}^{0} \equiv \hat{K}^{0}\left(E_{m}\right), \quad K_{m}^{01} \equiv K^{01}\left[E_{m},(-1)^{m} \lambda\right], \\
Z_{ \pm m} \equiv & \operatorname{det}\left\{\hat{K}_{ \pm m}^{0}+K_{ \pm m}^{01}+\Pi^{S, P}\left[E_{ \pm m},(-1)^{m} \lambda\right]\right\}, \quad m>0
\end{aligned}
$$

we rewrite Eqs. (30) in an expanded form, explicitly performing the recurrences for the functions $P$ and $S$. The result can be presented in the form of the series for the functions $\Pi$ :

$$
\begin{gathered}
\hat{\Pi}^{S}=\sum_{m=1}^{\infty} \hat{\Pi}_{m}^{S}, \quad \Pi^{S 1}=\sum_{m=1}^{\infty} K_{m}^{01} \prod_{\alpha=1}^{m} \tilde{M}_{\alpha-1 / 2} Z_{\alpha}^{-1}, \\
\hat{\Pi}_{m}^{S}=G_{+0} G_{1}, \ldots, G_{m-1} \hat{K}_{m}^{0} G_{m-1}, \ldots, G_{1} G_{+0} \prod_{\alpha=1}^{m} Z_{\alpha}^{-1} .
\end{gathered}
$$

The series for $\Pi^{P}$ differ from Eqs. (45) by opposite signs of all lower indices. With this remark, the series for the functions $P$ and $S$ can be obtained from Eqs. (45) and (44). These series can be interpreted as asymptotic expansions over $\gamma^{-1}$, due to the presence of large parameter $\gamma \gg 1$ in $Z_{\alpha}$. Physically, these expansions reflect the nature of the net current as a sum of $n$-particle tunnel currents; ${ }^{4}$ each of them exists at $e V>2 \Delta / n$ and scales as $\gamma^{1-n}$ with respect to the single-particle current. The latter fact allows us to consider the $n$-particle current only within its actual voltage region $2 \Delta / n<e V<2 \Delta /(n-1)$; at larger voltages, the $(n-1)$-particle current dominates. Estimation shows that $m$ th terms in Eqs. (45) contribute to the $(m+$ 1)-particle current; thus, it is enough to consider them only at $e V<2 \Delta / m$, which greatly simplifies the structure of the series.

Indeed, consider the term $\hat{\Pi}_{1}^{S}$ proportional to the product

$$
G_{+0} \hat{K}_{1}^{0} G_{+0}=-\tilde{M}_{+} \hat{K}_{1}^{0}-\frac{1}{2} N_{\alpha} M_{\beta}^{2}\left(f_{\alpha}-f_{\beta}\right) \tau_{+},
$$

where $\alpha=3 / 2, \beta=1 / 2$, and we used the identities $F_{\alpha}^{2}=$ 1 and $F_{\alpha} F_{\beta} F_{\alpha}=\tau_{z}+\left(4 f_{\alpha}-2 f_{\beta}\right) \tau_{+}$. The allowed energy region determined by the last term in Eq. (46) is similar to that for the function $L_{\alpha \beta}$ [see Eqs. (37) and (38)]; therefore, this term turns to zero at $e V<2 \Delta$, according to Eq. (39). Thus, in this voltage region, the action of matrix envelopes $G_{+0}$ on the matrix $\hat{K}_{1}^{0}$ is reduced to multiplication on the scalar factor $-\widetilde{M}_{+}$. Similar considerations applied to each term of the expansion (45a) lead to the following simplified series for the functions $\hat{\Pi}$ :

$$
\hat{\Pi}^{S}=\sum_{m=1}^{\infty} \theta(2 \Delta-m e V)(-1)^{m} \hat{K}_{m}^{0} \prod_{\alpha=1}^{m} \tilde{M}_{\alpha,-1 / 2} Z_{\alpha}^{-1},
$$

where we introduced the Heaviside step function $\theta$ to specify explicitly the relevant voltage regions. The series for the functions $\hat{\Pi}^{P}$ differ from Eq. (47) by opposite signs of lower indices.

Now we proceed with asymptotic evaluation of the dc current. First we consider the contribution $j_{0}$ in Eq. (35a) to the net current spectral density which, according to Eq. (34), can be presented as a sum of three terms

$$
\begin{gathered}
j_{0}=j_{0}^{K}+j_{0}^{P}+j_{0}^{S}, \\
j_{0}^{K}=-\frac{2}{Z_{K}}\left[2 K_{0}^{z}\left(N_{+} f_{+}-N_{-} f_{-}\right)-K_{0}^{+}\left(N_{+}-N_{-}\right)\right] .
\end{gathered}
$$

The first term is equal to $-\left(4 / Z_{K}\right) L_{1 / 2,-1 / 2}$ and represents the spectral density of the single-particle current. According to Eqs. (38) and (39), it exists within the energy interval $|E|<-\Delta+e V / 2$ and vanishes at $e V<2 \Delta$. Thus, at subgap voltages, we have to involve the terms $j_{0}^{P, S}$, which differ from Eq. (49) by replacements $K_{0} \rightarrow \Pi^{P, S}$. Considering, e.g., the spectral density $j_{0}^{S}$ and taking into account Eq. (47), we found that the $m$ th term in the expansion of $j_{0}^{S}$ is proportional to

$$
\theta(2 \Delta-m e V) \sum_{\alpha, \beta= \pm 1 / 2} \beta L_{m+\alpha, \beta} .
$$

As follows from Eq. (39), only the term with $\alpha=-\beta=1 / 2$, proportional to $\theta[(m+1) e V-2 \Delta]$, survives in Eq. (50). Thus, we obtain the following series for $j_{0}^{S}$ :

$$
\begin{gathered}
j_{0}^{S}=\frac{2}{Z_{K}} \sum_{m=2}^{\infty} \chi_{m}(V) L_{m-1 / 2,-1 / 2} \prod_{\alpha=1}^{m-1} \frac{a_{\alpha-1 / 2}}{\left|Z_{\alpha}\right|}, \quad a_{\alpha}=\frac{\bar{M}_{\alpha}^{2}}{4} \\
\chi_{m}(V)=\left\{\begin{array}{cc}
1 & 2 \Delta / m<e V<2 \Delta /(m-1), \\
0 & \text { otherwise. }
\end{array}\right.
\end{gathered}
$$

By applying similar considerations to $j_{0}^{P}$ and $j_{1}+j_{-1}$, we arrive at the formula for full generalized current spectral density [at $n=1$, the product in Eq. (53) is unity]

$$
\begin{gathered}
j(E, \lambda)=\sum_{n=1}^{\infty} \chi_{n}(V) j^{(n)}, \\
j^{(n)}=\frac{4}{\left|Z_{K}\right|} \sum_{m=1}^{n} L_{m-1 / 2, m-n-1 / 2} \prod_{\alpha=m-n+1}^{m-1} \frac{a_{\alpha-1 / 2}}{\left|Z_{\alpha}\right|} .
\end{gathered}
$$

According to Eq. (53), the $n$-particle spectral density $j^{(n)}$ consists of $n$ equal contributions of MAR chains with $n$ steps. Each chain starts at the energy $E_{m-n-1 / 2}<-\Delta$ and finishes at $E_{m-1 / 2}>\Delta$, thus transferring the quasiparticles to the extended states above the energy gap, which results in the formation of the dissipative current. The intermediate energies $E_{\alpha-1 / 2}$ inside the gap correspond to the points of Andreev reflections. These contributions are nonzero within the energy intervals of width $n e V-2 \Delta$, which are distributed 
equidistantly (with spacing $e V$ ) along the energy axis and symmetrically with respect to the zero energy, in conformity with the numerical results shown in Fig. 3. This enables us to write down the full dc current as a sum of $n$-particle tunnel currents $I^{(n)}$, where only one term in $j^{(n)}$ multiplied by $n$ is taken into account:

$$
\begin{gathered}
I=\sum_{n=1}^{\infty} \chi_{n}(V) I^{(n)}, \\
I^{(n)}=n \int_{-\infty}^{\infty} \frac{d \lambda}{2 \pi} \int_{\Delta-e V / 2}^{-\Delta+(n-1 / 2) e V} \frac{d E}{e R} \frac{N_{1 / 2} N_{1 / 2-n}}{\left|Z_{K}\right|} \prod_{\alpha=1}^{n-1} \frac{a_{1 / 2-\alpha}}{\left|Z_{-\alpha}\right|},
\end{gathered}
$$

At $n=1$, the product in Eq.(55) is assumed to be unity.

To complete our consideration, we present final expressions of $n$-particle currents for $n=1,2$, and 3 obtained from Eq. (55) by the integration over $\lambda$. A nontrivial point in this procedure is a proper choice of approximation for the determinants $Z$. In the single-particle current, it is enough to take $Z_{K}$ in the main approximation as det $K^{0}=-\left[\left(\lambda-\tau_{d} E\right)^{2}+(1 / 4)\left(N_{+}+N_{-}\right)^{2}\right]$, neglecting contributions of $\Pi^{P, S}$ to the function $\widetilde{K}$ in Eq. (34). As is obvious from this expression, the parameter $\tau_{d}$ drops out from $I^{(1)}$, and we obtain a simple formula

$$
I^{(1)}=\int_{\Delta-\frac{e V}{2}}^{-\Delta+\frac{e V}{2}} \frac{d E}{e R\left(N_{+}^{-1}+N_{-}^{-1}\right)} .
$$

From the standpoint of the circuit theory for incoherent SINIS structures, ${ }^{16}$ this result can be interpreted as the Ohm's law for two tunnel resistors $R_{ \pm}=R N_{ \pm}^{-1}$ connected in series.

In calculation of the two-particle current, the main approximation is applicable to the determinant $Z_{-1}=-\left|X_{-1}\right|^{2}$, whereas in $Z_{K}=-\left|X_{K}\right|^{2}$, one should hold the term $\Pi^{P}$ :

$$
\begin{gathered}
X_{-1}=i \lambda_{-1}+\frac{1}{2} N_{-3 / 2}, \quad X_{K}=i \lambda_{K}+\frac{1}{2} N_{+}+\frac{a_{-}}{X_{-1}}, \\
\lambda_{K}=\lambda-\tau_{d} E+\frac{1}{2} \bar{N}_{-}, \quad \lambda_{-1}=-\lambda_{K}-2 \tau_{d} E_{-}+\bar{N}_{-} .
\end{gathered}
$$

By taking $\lambda_{K}$ as a new integration variable, we see that its characteristic value is of the order of unity, which enables us to approximate $\lambda_{-1}$ as $-2 \tau_{d} E_{-}$. After integration over $\lambda_{K}$ and symmetrization of the allowed energy interval, we obtain

$$
I^{(2)}=\int_{\Delta-e V}^{-\Delta+e V} \frac{d E}{e R} \frac{8 N_{1} a N_{-1}}{N_{1}\left[\left(4 \tau_{d} E\right)^{2}+N_{-1}^{2}\right]+4 a N_{-1}} .
$$

The current spectral density in Eq. (57) has a resonant form, with a sharp peak at zero energy (the resonant nature of the even-particle currents has been already noted in Sec. III). If the applied voltage is not very close to the threshold $\Delta / e$ of the two-particle current, the integral in Eq. (57) can be calculated in the resonant approximation by assuming $E=0$ in all spectral functions and spreading the limits to $\pm \infty$,

$$
I^{(2)}=\frac{\pi \Delta}{2 \gamma e R} \frac{N(e V)}{\sqrt{1+N^{2}(e V)}} \text {. }
$$

Similar considerations lead to the following expression for the three-particle current:

$$
I^{(3)}=\frac{3}{4 \gamma^{2} e R} \int_{\Delta-\frac{3}{2} e V}^{-\Delta+\frac{3}{2} e V} \frac{d E N_{\frac{3}{2}} a_{+} a_{-} N_{-\frac{3}{2}}}{a_{-} N_{-\frac{3}{2}} E_{+}^{2}+a_{+} N_{\frac{3}{2}} E_{-}^{2}}
$$

Expressions (56)-(59) well reproduce the results of full numerical calculations for large $\gamma$ shown in Figs. 1 and 2.

\section{SUMMARY}

We have calculated, both numerically and analytically, the current-voltage characteristics (IVCs) of a diffusive SINIS junction, where $S$ are local-equilibrium superconducting reservoirs, I denotes tunnel barriers, and $\mathrm{N}$ is a short diffusive normal wire, the length of which is much smaller than the coherence length and the resistance $R_{N}$ is much smaller than the resistance $R$ of the tunnel barriers. The regime of coherent MAR transport in such structure is governed by the parameter $\gamma=\tau_{d} \Delta$, which represents a characteristic phase shift between the wave functions of the electron and the retroreflected hole accumulated during the quasiparticle dwell time $\tau_{d} \sim E_{\mathrm{Th}}^{-1}\left(R / R_{N}\right)$. We demonstrated that the Keldysh-Green's function equations for this problem can be efficiently solved in the whole range of electron-hole dephasing $0<\gamma<\infty$. This is achieved by reducing the solution of full $4 \times 4$ matrix two-time Keldysh-Green's function equations $^{14}$ to the solution of the $2 \times 2$ matrix recurrence relations of the second order, similar to the recurrences in analogous ballistic problems. ${ }^{4,5}$ In the limit of small dephasing $\gamma \rightarrow 0$, our solution reduces to a known formula for mesoscopic connector, ${ }^{24}$ i.e., averaging of the result for the single-channel junction over the distribution of transparencies for the corresponding double-barrier normal diffusive structure. $^{9}$

In the opposite case of large electron-hole dephasing $\gamma \gg 1$, the subharmonic gap structure in the IVC scales with $\gamma^{-1}$; this means that the $n$-particle tunnel currents scale as $\gamma^{1-n}$ with respect to the single-particle current, and $\gamma^{-1}$ plays the role of an effective tunneling parameter. Due to the presence of resonant MAR chains touching the edges of small minigap $\Delta_{g} \approx \Delta /(\gamma+1)$, the even subharmonics are enhanced, and corresponding portions of the IVC show negative differential resistance. We presented analytical results for the excess current at arbitrary $\gamma$ and for multiparticle currents at $\gamma \gg 1$.

For experimental observation of the phenomena discussed in this paper, the most stringent constraint concerns the Josephson regime. This implies small values of the dwell time compared to the inelastic relaxation time. If this requirement is not fulfilled, the central metallic island acts as a reservoir, and the structure splits in two NIS junctions connected in series. This is the case of SINIS junctions extensively used in microcoolers ${ }^{32}$ and SET turnstiles. ${ }^{33}$ For the Josephson effect to occur in metallic SINIS junctions with conventional oxide tunnel barriers, a sandwich-type junction must be employed having extremely thin normal metallic layer not exceeding $10 \mathrm{~nm}$. Such junctions have been developed using $\mathrm{Nb} / \mathrm{AlO}_{x} / \mathrm{Al} / \mathrm{AlO}_{x} / \mathrm{Nb}$ technology, and they demonstrated 
rather large values of $\gamma \sim 10^{4}$ and a pronounced deficit current. ${ }^{34-36}$ This is precisely the limit of large electron-hole dephasing studied in this paper. In order to investigate a crossover to the regime of small dephasing at $\gamma \sim 1$, one needs to use junctions with more transparent NS interfaces, such as junctions based on diffusive InAs two-dimensional electron gas or graphene, or corresponding nanowires and nanotubes. *eugene.bezuglyi@gmail.com

${ }^{1}$ T. M. Klapwijk, G. E. Blonder, and M. Tinkham, Physica B+C 109-110, 1657 (1982).

${ }^{2}$ G. B. Arnold, J. Low Temp. Phys. 68, 1 (1987).

${ }^{3}$ U. Gunsenheimer and A. D. Zaikin, Phys. Rev. B 50, 6317 (1994).

${ }^{4}$ E. N. Bratus', V. S. Shumeiko, and G. Wendin, Phys. Rev. Lett. 74, 2110 (1995).

${ }^{5}$ D. Averin and A. Bardas, Phys. Rev. Lett. 75, 1831 (1995).

${ }^{6}$ J. C. Cuevas, A. Martín-Rodero, and A. Levy Yeyati, Phys. Rev. B 54, 7366 (1996).

${ }^{7}$ A. Bardas and D. V. Averin, Phys. Rev. B 56, R8518 (1997).

${ }^{8}$ Y. Naveh, Vijay Patel, D. V. Averin, K. K. Likharev, and J. E. Lukens, Phys. Rev. Lett. 85, 5404 (2000).

${ }^{9}$ A. Brinkman and A. A. Golubov, Phys. Rev. B 61, 11297 (2000).

${ }^{10} \AA$ A. Ingerman, G. Johansson, V. S. Shumeiko, and G. Wendin, Phys. Rev. B 64, 144504 (2001).

${ }^{11}$ A. Levy Yeyati, J. C. Cuevas, A. López-Dávalos, and A. MartínRodero, Phys. Rev. B 55, R6137 (1997).

${ }^{12}$ G. Johansson, E. N. Bratus', V. S. Shumeiko, and G. Wendin, Phys. Rev. B 60, 1382 (1999).

${ }^{13}$ P. Samuelsson, G. Johansson, Å. Ingerman, V. S. Shumeiko, and G. Wendin, Phys. Rev. B 65, 180514(R) (2002).

${ }^{14}$ A. I. Larkin and Yu. N. Ovchinnikov, in Nonequilibrium Superconductivity, edited by D. N. Langenberg and A. I. Larkin (Elsevier, Amsterdam, 1986).

${ }^{15}$ A. V. Zaitsev and D. V. Averin, Phys. Rev. Lett. 80, 3602 (1998).

${ }^{16}$ E. V. Bezuglyi, E. N. Bratus', V. S. Shumeiko, G. Wendin, and H. Takayanagi, Phys. Rev. B 62, 14439 (2000).

${ }^{17}$ E. V. Bezuglyi, E. N. Bratus', V. S. Shumeiko, and G. Wendin, Phys. Rev. B 63, 100501(R) (2001).

${ }^{18}$ M. Octavio, M. Tinkham, G. E. Blonder, and T. M. Klapwijk, Phys. Rev. B 27, 6739 (1983).

${ }^{19}$ E. V. Bezuglyi, A. S. Vasenko, E. N. Bratus', V. S. Shumeiko, and G. Wendin, Phys. Rev. B 73, 220506 (2006).
${ }^{20}$ J. C. Cuevas, J. Hammer, J. Kopu, J. K. Viljas, and M. Eschrig, Phys. Rev. B 73, 184505 (2006).

${ }^{21}$ R. Seviour and A. F. Volkov, Phys. Rev. B 61, 9273 (2000),

${ }^{22}$ A. Brinkman, A. A. Golubov, H. Rogalla, F. K. Wilhelm, and M. Yu. Kupriyanov, Phys. Rev. B 68, 224513 (2003).

${ }^{23}$ E. V. Bezuglyi, V. S. Shumeiko, and G. Wendin, Phys. Rev. B 68, 134506 (2003).

${ }^{24}$ Yu. V. Nazarov, Superlattices Microstruct. 25, 1221 (1999); W. Belzig and Yu. V. Nazarov, Phys. Rev. Lett. 87, 197006 (2001).

${ }^{25}$ J. A. Melsen and C. W. J. Beenakker, Physica B 203, 219 (1994); W. Belzig, A. Brataas, Yu. V. Nazarov, and G. E. W. Bauer, Phys. Rev. B 62, 9726 (2000).

${ }^{26}$ M. Yu. Kupriyanov and V. F. Lukichev, Sov. Phys. JETP 67, 1163 (1988) [Zh. Eksp. Teor. Fiz. 94, 139 (1988)].

${ }^{27}$ P. Samuelsson, Phys. Rev. B 67, 054508 (2003).

${ }^{28}$ J. Börlin, W. Belzig, and C. Bruder, Phys. Rev. Lett. 88, 197001 (2002).

${ }^{29}$ K. M. Schep and G. E. W. Bauer, Phys. Rev. Lett. 78, 3015 (1997); Phys. Rev. B 56, 15860 (1997).

${ }^{30}$ E. N. Bratus', V. S. Shumeiko, E. V. Bezuglyi, and G. Wendin, Phys. Rev. B 55, 12666 (1997).

${ }^{31}$ V. S. Shumeiko, E. N. Bratus', and G. Wendin, Low Temp. Phys. 23, 181 (1997).

${ }^{32}$ F. Giazotto, T. T. Heikkilä, A. Luukanen, A. M. Savin, and J. P. Pekola, Rev. Mod. Phys. 78, 217 (2006).

${ }^{33}$ J. P. Pekola, V. F. Maisi, S. Kafanov, N. Chekurov, A. Kemppinen, Yu. A. Pashkin, O.-P. Saira, M. Möttönen, and J. S. Tsai, Phys. Rev. Lett. 105, 026803 (2010).

${ }^{34}$ E. Bartolomé, A. Brinkman, J. Flokstra, A. A. Golubov, and H. Rogalla, Phys. C (Amsterdam) 340, 93 (2000).

${ }^{35}$ M. M. Th. M. Dierichs, P. Dieleman, J. J. Wezelman, C. E. Honingh, and T. M. Klapwijk, Appl. Phys. Lett. 64, 921 (1994).

${ }^{36}$ I. P. Nevirkovets, J. B. Ketterson, and S. Lomatch, Appl. Phys. Lett. 74, 1624 (1999). 